\title{
Versuche über die gegenseitige funktionelle Beeinflussung von Gross- und Kleinhirn.
}

\author{
Von \\ Prof. Dr A. Beck und Prof. Dr. G. Bikeles.
}

(Mit 2 Textfiguren.)

\footnotetext{
Bonn, 1911.

S॰parat-Abdruck aus dem Archiv für die ges. Physiologie Bd. 143.

Verlag von Martin Hager.
} 


$$
H-121545
$$

http://rcin.org.pl/ifis 


\section{PAN 11349}
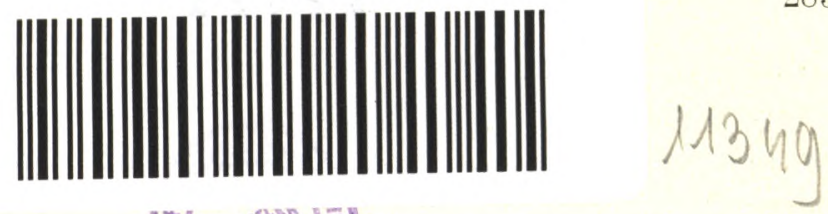

(Aus dem physiologischen Institut der Universität Lemberg.)

\section{Versuche über die gegenseitige funktionelle Beeinflussung von Gross- und Kleinhirn ${ }^{1}$ ).}

Von

Prof. Dr. A. Beck und Prof. Dr. G. Bikeles.

(Mit 2 Textfiguren.)

Ohne hier auf die verschiedenen Theorien über das Wesen der Kleinhirnfunktion überhaupt ${ }^{2}$ ) einzugehen, wollen wir die Ergebnisse einer, so weit uns bekannt, in dieser Frage bis nun nicht zur Anwendung gekommenen Untersuchungsmethode mitteilen. Wir meinen nämlich das Verfahren des Nachweises von Aktionsströmen, entstanden im Kleinhirn bei Reizung des Grosshirns und umgekehrt.

$\mathrm{Zu}$ diesem Zwecke stellten wir zunächst Vorversuche mittels elektrischer Reizung sei es des Grosshirns, sei es des Kleinhirns an. Jedoch überzeugten wir uns alsbald, dass auf diese Weise irgendwie sichere Resultate infolge von in das Galvanometer übergehenden Stromschleifen gar nicht zu erwarten sind.

Wir sahen uns daher gezwungen, ein anderes - wie die vielfachen nachfolgenden Untersuchungen zeigten - sicheres Verfahren einzuschlagen.

Die Grosshirn- bezüglich Kleinhirnrinde wurde nicht elektrisch, sondern thermisch gereizt mit Temperaturen, von etwa $55-58^{\circ}$ C. und zwar in der Weise, dass die zu reizende Stelle der Rinde mit dem stumpfen Ende einer mit zu- und abfliessendem warmem Wasser gespeisten nach der Art eines Thermästheriometers

1) Vorgelegt der Krakauer Akademie der Wissenschaften in der Sitzung vom 6. Oktober 1911, erscheint in polnischer Sprache in den Abhandlungen der Akademie Bd̀. 51 B.

2) Da wir mehrfache Versuche, betreffend die Funktion des Kleinhirns, vorhaben, gehen wir in diesen Arbeiten auf die Literatur vorläufig nicht ein. 
konstruierten Doppelröhre vorsichtig durch etwa 1-2 Sek. berührt wurde. Dabei wurde zur möglichsten Vermeidung einer Alteration der Rinde dafür gesorgt, dass innerhalb eines gewissen für die Untersuchung gewählten Bezirkes, verschiedene Stellen abwechselnd gereizt werden. Wiederholt gereizt wurde eine und dieselbe Stelle der Rinde nur nach einer längeren Pause.

In manchen Versuchen zeigte sich nach etwas länger stattgehabten Reizapplikationen Hyperämie eventuell leichtes Ödem der auf obige Weise gereizten Rindenpartie; natürlich wurde bei dem Eintreten der geringsten sichtbaren Veränderung jede weitere Untersuchung eingestellt.

Häufig und überwiegend behielt aber die gereizte Rindenpartie auch bei länger fortgesetzter Untersuchung ihr gewöhnliches Aussehen, und es gelang uns sogar wiederholt nachfolgend von einer solchen Stelle der Grosshirnrinde aus bei Reizung des zentralen Stumpfes eines entsprechenden peripheren Nerven Aktionsströme zu erhalten von einer Intensität, wie von einer eben erst blossgelegten Hirnrinde.

Die hier mitzuteilenden Versuche zerfallen in zwei Serien:

In der einen Serie wurde thermisch die Grosshirnrinde gereizt, vor allem in der psychomotorischen Region, häufig aber auch zum Vergleich die hinter dem Gyrus sigmoideus befindliche Gegend. (Vgl. das Schema Fig. 1.) Abgeleitet wurde dann von der Kleinhirnrinde, vor allem der kontralateralen Hemisphäre, sehr oft auch von der gleichseitigen Hemisphäre und in manchen Versuchen zum Vergleich auch vom Vermis posterior (Lobus medianus posterior nach Bolk).

In der zweiten Serie wurde thermisch die Kleinhirnrinde (Hemisphäre) gereizt. Abgeleitet wurde dann von der kontralateralen eventuell auch der gleichseitigen Grosshirnrinde, vor allem wieder von der psychomotorischen Region, zum Vergleich aber auch von einem hinter der psychomotorischen Region gelegenen Bezirk.

Da zum Nachweis von Aktionsströmen, das ist der Negativität einer bestimmten Rindenstelle, es notwendig ist, eine der unpolarisierbaren Elektroden auf eine indifferente nicht in Aktionszustand geratende Gegend zu setzen, wählten wir dazu in beiden Versuchsserien einen Punkt am Hinterhauptslappen kontralateral von der blossgelegten psychomotorischen Region. 
Das spezielle Vorgehen und die Versuchsanordnung in beiden Serien war folgende: Am curaresierten Hunde wurde einerseits, in der Regel links, die psychomotorische Region und noch dazu ein bedeutender Bezirk hinter derselben (Fig. 1 horizontale Schraffierung) blossgelegt. Auf der zweiten Seite (gewöhnlich rechts) wiederum wurde am Hinterhauptslappen eine runde etwa $2 \mathrm{~cm}$ im Durchmesser betragende Stelle freigelegt. Darauf wurde die hintere Oberfläche des Kleinhirns, d, i. Vermis poster. (Lob. medianus post.) und beide hinteren Hemisphärenabschnitte bis zum Tentorium blosspräpariert. Selbstverständlich wurde bei der Blosslegung ängstlich darauf geachtet, dass die Rinde vollständig unversehrt blieb.

Von den unpolarisierbaren Elektroden, deren Enden dicke mit Ringer'scher Lösung durchtränkte Wollfälen bildeten, wurde hierauf die eine an den Hinterhauptslappen als indifferente Stelle, die andere je nach dem Versuchszweck an die zu untersuchende Stelle der Gross- resp. Kleinhirnrinde angelegt. Die primäre $\mathrm{Ab}$ lenkung des auf diese Weise mit der Rinde verbundenen Galvanometers wurde nun vor jedem (thermischen) Reizversuch abgelesen und notiert. Ebenfalls wurde

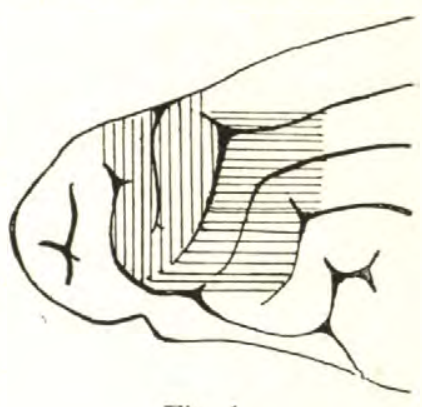

Fig. 1. die während der jedesmaligen stattgehabten Reizung erfolgende Galvanometerschwankung, wie diejenige unmittelbar nach der Reizung, abgelesen und notiert.

Der auf diese Weise konstatierte Unterschied (in der Richtung) der Galvanometerschwankung während der Reizdauer galt für uns nur dann als Ausdruck von infolge der Reizung auftretenden Aktionsströmen, wenn die entsprechende für die funktionelle gegenseitige Abhängigkeit in Frage kommende Rindenstelle des Gross- resp. Kleinhirns elektronegativ wurde. In unseren im weiteren anzuführenden Ergebnissen bezeichen wir eine derartige Galvanometerschwankung mit dem Zeichen - bei der Zahl, welche die Grösse des Ausschlages in Skalenteilen ausdrückt.

Als negativ bezeichnen wir aber nur solche während der Reizdauer erfolgten Ablenkungen, bei denen das Galvanometer unmittelbar nach der Reizung in der Richtung zur primären Ablenkung zurückkehrte. Falls aber eine solche Rückkehr nicht statthatte, sei 
es, dass das Galvanometer auf derselben Stelle wie am Ende der Reizung beharrte, sei es in derselben Richtung weiterging, dann bezeichnen wir die betreffenden wäbrend der Reizung abgelesenen Galvanometerschwankungen als in ihrer Bedeutung zweifelhaft mit einem Fragezeichen (?). Das Ausbleiben einer Galvanometerablenkung während der Reizung, ebenso jede erfolgte Ablenkung von einer Richtung, welche zeigte, dass die in Frage kommende Rindenregion elektropositiv wurde, bezeichnen wir mit Null.

\section{Serie.}

\section{Versuche mittels Reizung der Grosshirmrinde und Ableitung von der Kleinhirnrinde.}

In 21 Versuchen wurde bei thermischer Reizung der in der Regel linksseitigen psychomotorischen Region von der kontralateralen (rechten) Kleinhirnhemisphäre und zwar sei es vom Lobulus paramedianus Bolk (Lobulus semilunaris inferior), sei es vom Crus secundum lobi ansiformis Bolk (Lobulus semilunaris sup.) abgeleitet. In 17 von denselben Versuchen wurden ausserdem auch von der gleichseitigen (linken) Kleinhirnhemisphäre und zwar von identischen Stellen wie an der kontralateralen abgeleitet.

Um den Erfolg der Ableitung von einer gegebenen Rindenstelle des Kleinhirns festzustellen, wurde die psychomotorische Region (wie bereits hervorgehoben an verschiedenen Stellen) mehrmals hintereinauder - im Durchschnitt etwa achtmal, manchmal mehr, seltener weniger - gereizt und auf das Auftreten von Aktionsströmen an der abgeleiteten Stelle geachtet. Dasselbe Verfahren wiederholte sich, so oft irgendeine Kleinhirnrindenpartie neuerlich mit dem Galvanometer verbunjen wurde.

Es zeigte sich nun, dass bei Ableitung von der kontralateralen und sehr häufig ebenfalls bei Ableitung von der gleichseitigen Kleinhirnhemisphäre sich bei der erwähnten Versuchsanordnung das Auftreten von Aktionsströmen in der entsprechenden Kleinhirnrindenregion aufs deutlichste nachweisen liess.

Natürlich erfolgte nicht ausnahmslos auf jede einzelne Reizung ein unzweifelhaftes Negativwerden der untersuchten Stelle; manchmal war der Erfolg einzelner Reizungen zweifelhaft, oder manchmal blieb die Reizung erfolglos, wie dies gewöhnlich bei den Versuchen über die elektrischen Erscheinungen im Zentralnervensystem zu be- 
Versuche über die gegenseitige funkt. Beeinflussung von Grosshirn etc. 287 obachten ist, im grossen und ganzen aber war das Erscheinen von Aktionsströmen ein prägnantes.

Alle unsere Versuche im einzelnen gedenken wir nicht hier anzuführen; wir begnügen uns damit, zur Illustration als Beispiele Auszüge von Versuchen als Übersichtstabelle anzugeben.

In dieser Übersichtstabelle (Tab. 1) sind die Gesamtergebnisse aller dieser Versuche auch dem Prozente nach angegeben. Man ersiebt daraus, dass bei dieser Versuchsanordnung die Aktionsströme auf der kontralateralen Kleinhirnhenisphäre meist prävalieren. Und zwar ist das Überwiegen an der kontralateralen Seite ein ausgesprochenes in neun (auf 17) Versuchen, nur geringfügig in zwei Versuchen.

Tabelle I.

Häufigkeit und Stärke der Aktionsströme bei Ableitung von der gleichseitigen und kontralateralen Kleinhirnhemisphäre. Reizung der linken psychomotorischen Region.

\begin{tabular}{|c|c|c|c|c|c|c|c|c|c|c|}
\hline \multirow{3}{*}{ 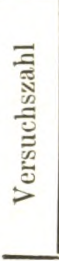 } & \multicolumn{5}{|c|}{$\begin{array}{c}\text { Ableitung von der rechten Klein- } \\
\text { hirnhemisphäre }\end{array}$} & \multicolumn{5}{|c|}{$\begin{array}{c}\text { Ableitung von der linken Klein- } \\
\text { hirnhemisphäre }\end{array}$} \\
\hline & \multirow{2}{*}{$\begin{array}{c}\text { Ge- } \\
\text { reizt } \\
\text { wurde } \\
\text { mal }\end{array}$} & \multicolumn{3}{|c|}{$\begin{array}{l}\text { Negativwerden des } \\
\text { Kleinhirns }\end{array}$} & \multirow{2}{*}{ 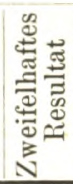 } & \multirow{2}{*}{$\begin{array}{c}\text { Ge- } \\
\text { reizt } \\
\text { wurde } \\
\text { mal }\end{array}$} & \multicolumn{3}{|c|}{$\begin{array}{l}\text { Negativwerden des } \\
\text { Kleinhirns }\end{array}$} & \multirow{2}{*}{ 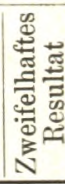 } \\
\hline & & mal & $\begin{array}{l}\text { in } \\
\text { Proz. }\end{array}$ & $\begin{array}{l}\text { Grösse der } \\
\text { Ablenkung } \\
\text { in S'kalent. }\end{array}$ & & & mal & $\begin{array}{l}\text { in } \\
\text { Proz. }\end{array}$ & $\begin{array}{c}\text { Grösse der } \\
\text { Ablenkung } \\
\text { in Skalent. }\end{array}$ & \\
\hline 1 & 5 & 4 & & $10-55$ & 1 & \multicolumn{5}{|c|}{ Nicht untersucht } \\
\hline 2 & 15 & 7 & 46,66 & $10-34$ & 2 & 10 & $3^{21}$ & 30 & $10-15$ & 1 \\
\hline$\overline{3}$ & 9 & 7 & 77,8 & $9-40$ & 1 & 8 & 4 & 50 & $5-12$ & 1 \\
\hline 4 & 7 & 6 & 85,7 & $7-13$ & 1 & 8 & 1 & & & 4 \\
\hline 5 & 10 & 7 & 70 & $9-22$ & 1 & 10 & 5 & 50 & $5-15$ & 1 \\
\hline 6 & 16 & 6 & 37,5 & $5-11$ & 4 & 10 & 8 & 80 & $5-10--16$ & \\
\hline 7 & 9 & 4 & 44,4 & $10-19$ & 1 & 10 & 5 & 50 & $4-12$ & \\
\hline 8 & 10 & 5 & 50 & $7-17$ & & 9 & 5 & 55,5 & $3-13$ & 1 \\
\hline 9 & 7 & 5 & 70 & $6,5-16$ & & 7 & 6 & 88,8 & $6,5-12$ & 1 \\
\hline 10 & 10 & 6 & 60 & $7-14$ & 1 & 10 & 0 & & & \\
\hline 11 & 6 & 4 & 83 & $5-13$ & 1 & \multicolumn{5}{|c|}{ Nicht untersucht } \\
\hline 12$\}$ & a) 9 & 6 & 67 & $8-16$ & 1 & 6 & 1 & & & \\
\hline$\left.{ }_{13}\right\rangle$ & b) $\begin{array}{l}9 \\
9\end{array}$ & $\begin{array}{l}5 \\
7\end{array}$ & $\begin{array}{l}56 \\
78\end{array}$ & $\begin{array}{r}4-10 \\
11-16\end{array}$ & $\begin{array}{l}2 \\
1\end{array}$ & 7 & 5 & 71 & $4-12$ & \\
\hline 14 & 8 & 7 & 87 & $\begin{array}{r}11-10 \\
9,5-12\end{array}$ & & 8 & 6 & 75 & $8-14,5$ & \\
\hline 15\{ & $\begin{array}{l}\text { a) } 6 \\
\text { b) } 7\end{array}$ & $\begin{array}{l}5 \\
5\end{array}$ & $\begin{array}{l}83 \\
71\end{array}$ & $\begin{array}{l}9-12 \\
6-8\end{array}$ & & \multicolumn{5}{|c|}{ Nicht untersucht } \\
\hline 16\{ & a) 8 & 5 & 62,5 & $12-15$ & 1 & 8 & 5 & 62,5 & $6,5-12$ & \\
\hline $17^{\prime}$ & b) $\stackrel{3}{8}$ & $\begin{array}{l}3 \\
4\end{array}$ & $\begin{array}{r}100 \\
50\end{array}$ & $\begin{array}{l}8-12 \\
6-8\end{array}$ & & 7 & 0 & & & \\
\hline 18 & 8 & 4 & 50 & $7-16$ & & 7 & 4 & 55 & $8-12$ & 2 \\
\hline 19 & 9 & 5 & 55,5 & $6-17,5$ & 1 & 8 & 6 & 75 & $5-14$ & 1 \\
\hline 20 & 6 & 6 & 100 & $7-11$ & & 8 & 6 & 75 & $5-8$ & 1 \\
\hline 21 & 5 & 4 & 80 & $4-7$ & & & & $t$ & परता & \\
\hline
\end{tabular}


Ein Ergebnis ohne wesentlichen Unterschied in der Zahl der erfolgreichen Reizungen bei Ableitung von der einen und der anderen Seite war in drei Versuchen zu konstatieren.

Hervorzuheben ist, dass in drei Versuchen (Versuchszahl 6, 9 und 19) die Aktionsströme auf der der Reizung gleichseitigen Kleinhirnhemisphäre und zwar in zwei in auffallender Weise häufiger als auf der kontralateralen auftraten.

Dies besagt in anderen Worten: Der Einfluss einer Grosshirnhemisphäre erstreckt sich auf beide Kleinhirnhemisphären und zwar in der Art, dass dieser Einfluss auf die kontralaterale Kleinhirnhemisphäre meist überwiegt. In einer Minorität der Fälle aber betrifft dieser zerebrale Einfluss beide Kleinhirnhemisphären ohne wesentlichen Unterschied; in manchen kann sogar das Verhältnis ein umgekehrtes sein, d. h. gerade die gleichseitige Kleinhirnhemisphäre wird dann in stärkerem Maasse als die kontralaterale beeinflusst.

Diese Konstatierungen bezüglich der funktionellen Verhältnisse stehen in gutem Einklang mit den anatomischen Tatsachen von einer nicht kompleten, sondern nur überwiegenden Kreuzung der ponto-zerebellaren Bahnen. Dass individuelle Variationen in dem Grad der Kreuzung vorkommen, darauf weisen die Angaben von Hitzig und Gudden (zitiert nach Mingazzini, Neurolog. Zentralbl. 1895, p. 658-659) hin; diesen beiden Autoren zufolge führt nämlich nicht immer Atrophie einer Grosshirnhemisphäre eine solche am Kleinhirn herbei, und manchmal soll die atrophische Kleinhirnhemisphäre nicht die kontralaterale, sondern die gleichseitige sein.

Hervorheben müssen wir an dieser Stelle, dass bei Reizung der Extremitätenregion der Hirnrinde und Ableitung von einer und derselben Stelle der Kleinhirnhemisphäre wir niemals irgendeinen Unterschied im Auftreten resp. Ausbleiben von Aktionsströmen, welch $r$ von dem Reizungsort abhängig wäre, beobachteten. Reizung der Region für die vordere oder für die hintere Extremität verursachte an derselben abgeleiteten Stelle des Kleinhirns das Auftreten von Aktionsströmen von gleicher Intensität und Häufigkeit.

Mit bekannten anatomischen Tatsachen ebenfalls gut übereinstimmend sind unsere Versuchsergebnisse bezüglich des differenten 
Verhaltens betreffend das Auftreten von Aktionsströmen bei Ableitung von der Kleinhirnhemisphäre und bei Ableitung vom Mittelstück während der thermischen Reizung derselben Bezirke der psychomotorischen Region. Das Vorgehen bei dieser Versuchsreihe war folgendes: Zuerst leiteten wir von einer Kleinhirnhemisphäre ab. Nach Konstatierung, dass an dieser Partie unter dem Einfluss thermischer Reizung der Grosshirnrinde Aktionsströme häufig zum Vorschein kommen, verbanden wir wiederum mit dem Galvanometer den hinteren Vermisabschnitt (L. med. post. Bolk). Endlich wurde hierauf abermals von einer Kleinhirnhemisphäre abgeleitet. Auf diese Weise erhielten wir eine sichere Grundlage zum Vergleich des zerebralen Einflusses auf Hemisphäre einerseits und auf das Mittelstück andererseits.

Das Endresultat in drei derartigen Versuchen war der Nachweis ausgesprochener und häufiger Aktionsströme in den Kleinhirnhemisphären sowohl vor als auch nach der eingeschalteten Ableitung vom Mittelstück bei Fehlen resp. sehr zweifelhaftem Auftreten von Aktionsströmen bei Ableitung vom Mittelstück selbst. Nur in einem Falle zeigten sich auch bei Ableitung vom Mittelstück Aktionsströme doch jedenfalls etwas geringer als von den Hemisphären (vgl. Tab. II).

$$
\text { Tabelle II. }
$$

Verhalten der Aktionsströme bei Ableitung von Kleinhirnhemisphäre und Mittelstück (Vermis). Reizung der linken psychomotorischen Region.

\begin{tabular}{|c|c|c|c|c|c|}
\hline \multirow{2}{*}{$\begin{array}{l}\text { Ver- } \\
\text { suchs- } \\
\text { zahl }\end{array}$} & \multicolumn{5}{|c|}{ Ablenkung bei Ableitung vom Kleinhirn, und zwar } \\
\hline & $\begin{array}{l}\text { von der } \\
\text { rechten Klein- } \\
\text { hirnhemisphäre }\end{array}$ & $\begin{array}{c}\text { von der } \\
\text { linken Klein- } \\
\text { hirnhemisphäre }\end{array}$ & vom Vermis & $\begin{array}{c}\text { nochn } \\
\text { der K } \\
\text { hemi }\end{array}$ & $\begin{array}{l}\text { als von } \\
\text { einhirn- } \\
\text { phäre }\end{array}$ \\
\hline 1 & $\begin{array}{r}-12 \\
-16 \\
-14 \\
-11 \\
0 \\
-11\end{array}$ & $\begin{array}{r}-11,5 \\
0 \\
-12 \\
-5 \\
0 \\
-11 \\
-4\end{array}$ & $\begin{array}{r}0 \\
0 \\
-4 \\
? \\
0 \\
0 \\
0\end{array}$ & rechten & $\begin{array}{c}-3,5 \\
-3,7 \\
? \\
-8 \\
0\end{array}$ \\
\hline 2 & $\begin{array}{r}0 \\
0 \\
-15 \\
-15 \\
-15 \\
-12 \\
-11,5 \\
? \\
-12 \\
-8 \\
-8,5\end{array}$ & & $\begin{array}{c}? \\
? \\
0 \\
0 \\
0 \\
-7,5 \\
-7,5\end{array}$ & linken & $\begin{array}{c}0 \\
-6,5 \\
0 \\
-10,5 \\
-8 \\
-6,5 \\
0 \\
-12\end{array}$ \\
\hline
\end{tabular}




\begin{tabular}{|c|c|c|c|c|c|}
\hline \multirow{2}{*}{$\begin{array}{c}\text { Ver- } \\
\text { suchs- } \\
\text { zahl }\end{array}$} & \multicolumn{5}{|c|}{ Ablenkung bei Ableitung vom Kleinhirn, und zwar } \\
\hline & $\begin{array}{l}\text { von der } \\
\text { rechten Klein- } \\
\text { hirnhemisphäre }\end{array}$ & $\begin{array}{l}\text { von der } \\
\text { linken Klein- } \\
\text { hirnhemisphäre }\end{array}$ & vom Vermis & $\begin{array}{l}\text { noch } \\
\text { der } \mathrm{r} \\
\text { hem }\end{array}$ & $\begin{array}{l}\text { mals von } \\
\text { leinhirn- } \\
\text { isphäre }\end{array}$ \\
\hline 3 & $\begin{array}{r}0 \\
-12 \\
? \\
-16 \\
-12 \\
? \\
0 \\
-\quad 7\end{array}$ & $\begin{array}{r}0 \\
-8 \\
? ? \\
-12 \\
? \\
-8 \\
-8\end{array}$ & $\begin{array}{c}0 \\
-7 \\
-9 \\
0 \\
-6 \\
0 \\
-6,5 \\
0\end{array}$ & linken & $\begin{array}{c}0 \\
0 \\
-10 \\
-7,5 \\
0 \\
-4 \\
-5\end{array}$ \\
\hline 4 & $\begin{array}{c}-7,5 \\
-10 \\
-9 \\
-8 \\
-7 \\
-11 \\
0 \\
-9,5 \\
-9 \\
-6\end{array}$ & & $\begin{array}{r}-5 \\
0 \\
-7 \\
0 \\
0\end{array}$ & linken & $\begin{array}{r}-5 \\
-6 \\
-8 \\
-6,5 \\
0 \\
-8 \\
? \\
-5\end{array}$ \\
\hline
\end{tabular}

Von grosser Wichtigkeit schien uns die Beantwortung der Frage, ob für das Auftreten von Aktionsströmen in der Kleinhirnrinde eine

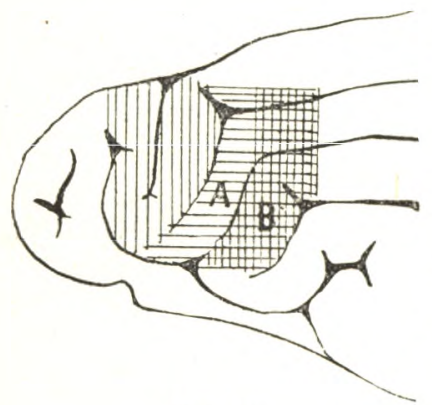

Fig. 2.

Reizung speziell nur an der psy'homotorischen Region wirksam sei. $\mathrm{Zu}$ diesem Zwecke wurde ausser der psychomotorischen Region auf derselben Seite ein ziemlich grosser Bezirk hinter der psychomotorischen Region (vgl. Schema) blossgelegt. Der Versuchsvorgang selbst war nun wie folgt: Abgeleitet wurde von der Kleinhirnhemisphäre. Thermisch gereizt wurde an der kontralateralen Grosshirnrinde die psychomotorische Region und zum Vergleich dẹr Bezirk hinter der psychomotorischen Region, und zwar häufig gesondert die an die psychomotorische Gegend unmittelbar angrenzende Partie (Fig. 2 $A$ ) und wiederum gesondert die weiter hinten gelegene Rindenpartie (Fig. $2 B$ ). Nach Möglichkeit wurde endlich abermals die psychomotorische Region gereizt.

In vier Versuchsfällen, bei denen das Ergebnis von Reizungen an der psychomotorischen Region zu Anfang und am Schluss dieses 
Versuchsverfahrens festgestellt werden kounte, zeigte sich die zwischen diese beiden erfolgreichen Reizungsreihen eingeschaltete Reizung besonders an dem weiter hinter dem Gyrus sigmoideus befindlichen Bezirk $(B)$ entweder ohne jedweden oder von ausgesprochen geringfügigem Erfolg. In zwei Versuchsfällen, wo eine Wiederholung der Reizung an der psychomotorischen Region nicht möglich war, konstatierten wir jedenfalls - übereinstimmend mit dem oben angeführten Resultat - einen bedeutend verringerten resp. minimalen Erfolg bei Reizung von der weiter hinter dem Gyrus sigmoideus gelegenen Rindenpartie $(B)$ im Vergleich zum Erfolge bei Reizung der psychomotorischen Region. In einem Versuch (Versuchszahl 3) wurde zum Vergleich nur die unmittelbar an den Gyrus sigmoideus angrenzende Partie $(A)$ gereizt; auch da ist die Intensität (und Häufigkeit) der erhaltenen $\Lambda$ ktionsströme geringer als bei Reizung der eigentlichen psychomotorischen Region (Tab. III).

$$
\text { T a belle III. }
$$

Vergleich der Reizungserfolge bei Reizung der psychomotorischen und der hinter derselben gelegenen Region. Ableitung von der rech ten Kleinhirnhemisphäre.

\begin{tabular}{|c|c|c|c|c|}
\hline \multirow{3}{*}{$\begin{array}{l}\text { Ver- } \\
\text { suchs- } \\
\text { zahl }\end{array}$} & \multicolumn{4}{|c|}{ Ablenkung bei Reizung der Grosshirnrinde, und zwar } \\
\hline & \multirow{2}{*}{$\begin{array}{l}\text { der psycho- } \\
\text { motorischen } \\
\text { Region }\end{array}$} & \multicolumn{2}{|c|}{ der linteren Rindenpartie } & \multirow{2}{*}{$\begin{array}{c}\text { nochmals der } \\
\text { psychomotorischer } \\
\text { Region }\end{array}$} \\
\hline & & $A$ & $B$ & \\
\hline 1 & $\begin{array}{r}0 \\
-12 \\
? \\
-16 \\
-12 \\
? \\
0 \\
-7\end{array}$ & & $\begin{array}{r}? \\
-5 \\
0 \\
? \\
0\end{array}$ & $\begin{array}{r}-7 \\
-8 \\
0 \\
-8 \\
? \\
-12 \\
-8 \\
-8\end{array}$ \\
\hline 2 & $\begin{array}{r}-12 \\
-9 \\
0 \\
-10,5 \\
-9,5 \\
-9,5\end{array}$ & $\begin{array}{r}0 \\
-9 \\
-9 \\
0 \\
-8\end{array}$ & $\begin{array}{r}-6 \\
-7 \\
0 \\
0 \\
0 \\
-7 \\
0\end{array}$ & $\begin{array}{r}0 \\
-8 \\
-7 \\
-7 \\
-6 \\
0 \\
-7\end{array}$ \\
\hline 3 & $\begin{array}{r}-12 \\
-11 \\
0 \\
-13 \\
-12 \\
-4 \\
-9,5 \\
-12,5\end{array}$ & $\begin{array}{r}0 \\
-7 \\
-7 \\
-8 \\
0\end{array}$ & & \\
\hline
\end{tabular}




\begin{tabular}{|c|c|c|c|c|}
\hline \multirow{3}{*}{$\begin{array}{l}\text { Ver- } \\
\text { suchs- } \\
\text { zahl }\end{array}$} & \multicolumn{4}{|c|}{ Ablenkung bei Reizung der Grosshirnrinde, und zwar } \\
\hline & \multirow{2}{*}{$\begin{array}{l}\text { der psycho- } \\
\text { motorischen } \\
\text { Region }\end{array}$} & \multicolumn{2}{|c|}{ der hinteren Rindenpartie } & \multirow{2}{*}{$\begin{array}{c}\text { nochmals der } \\
\text { psychomotorischen } \\
\text { Region }\end{array}$} \\
\hline & & $A$ & $B$ & \\
\hline 4 & $\begin{array}{l}-7,5 \\
-10 \\
-9 \\
-8 \\
-7 \\
-11 \\
-0 \\
-9,5 \\
-9 \\
-6\end{array}$ & $\begin{array}{l}-8 \\
-4 \\
-5,5 \\
-4,5\end{array}$ & $\begin{array}{r}0 \\
0 \\
-6\end{array}$ & \\
\hline 5 & $\begin{array}{r}0 \\
0 \\
-15 \\
-15 \\
-15 \\
-12 \\
-11,5 \\
?\end{array}$ & $\begin{array}{r}-5 \\
0\end{array}$ & $\begin{array}{l}0 \\
? \\
0\end{array}$ & \\
\hline 6 & $\begin{array}{r}-4 \\
-6 \\
0 \\
-7 \\
-7\end{array}$ & $\begin{array}{r}-6 \\
0 \\
?\end{array}$ & & $\begin{array}{c}? \\
-4 \\
-3 \\
-5 \\
-2,5 \\
?\end{array}$ \\
\hline 7 & $\begin{array}{r}-12 \\
-16 \\
-14 \\
-11 \\
0 \\
-11\end{array}$ & & $\begin{array}{l}0 \\
0 \\
0 \\
0 \\
0\end{array}$ & $\begin{array}{c}-10 \\
-4 \\
? \\
-11,5 \\
0 \\
-12 \\
-5 \\
0 \\
-11 \\
-4\end{array}$ \\
\hline
\end{tabular}

Diese Ergebnisse weisen darauf hin, dass wenigstens beim Hunde vor allem die psychomotorische Region es ist, welche vermittels der Brückenarme mit den Kleinhirnhemisphären in funktioneller Verbindung steht.

Wir glauben diese Eruierung - die sich auf dem Wege anatomischer Untersuchung, da es sich um unterbrochene Bahnen handelt, nicht leicht durchführen lässt - besonders hervorheben zu dürfen und weisen zugleich darauf hin, dass beim Hunde die psychomotorische Region auch Fühlsphäre und für den Muskelsinn von 
Versuche über die gegenseitige funkt. Beeinflussung von Grosshirn etc. 293

grosser Bedeutung ist ${ }^{1}$ ). Wir behalten uns vor, in weiteren Arbeiten über Kleinhirn, die wir vorhaben, auf diese Konstatierung und deren etwaige Bedeutung für die Kleinhirnfunktion zurückzukommen.

\section{Serie.}

\section{Versuche mittels Reizung der Kleinhirurinde und Ableitung von der Grosshirnrinde.}

In elf Versuchen wurde die Rinde der Kleinhirnhemisphäre thermisch gereizt bei Ableitung von Grosshirnrinde. In allen diesen Versuchen waren es mehr oder weniger identische Stellen der Kleinhirnhemisphäre, welche gereizt wurden und zwar am Lobulus paramedianus Bolk (Lobulus semilunaris inf.) und am Crus secundum Lobuli ansiformis Bolk (Lobulus semilunaris sup.), also an Bezirken, in denen bei Reizung der psychomotorischen Region der Grosshirnrinde Aktionsströme deutlichst beobachtet worden waren. Gereizt wurde in dieser Serie die Kleinhirnhemisphäre sowohl gleichseitig als auch kontralateral zu der abgeleiteten Grosshirnhemisphäre.

Das Ergebnis war aber hier im grossen und ganzen ein sehr abweichendes von dem in der Serie I. Nur in drei Versuchen gelang es bei diesem Versuchsverfahren Aktionsströme von einer Intensität und Häufigkeit annähernd wie bei Serie I nachzuweisen, während in acht Versuchen, also in der weit überwiegenden Mehrzahl, die eventuell zum Vorschein gekommenen negativen Galvanometerablenkungen von sehr geringer Häufigkeit waren (Tab. IV).

Diese so auffällige Differenz im Erhalten von Aktionsströmen zwischen beiden Versuchsanordnungen (Serie I und II) suggeriert förmlich die Annahme, dass beim Hunde wenigstens das Zuströmen

1) In einem Falle von gekreuzter Kleinhirnatrophie beim Menschen mitgeteilt von André Thomas und G. Cornelius (Revue neurol. 1907, nach Ref. im Neurolog. Zentralbl. 1908 S. 170) betrafen die ausgesprochensten Veränderungen an der Grosshirnrinde, den Stirnlappen, Parietallappen und einen Teil der Schläfenwindung, während die motorische Region wenig betroffen war. Dies könnte also gegen einen innigeren Zusammenhang zwischen psychomotorischer Region und Kleinhirnhemisphäre geltend gemacht werden. Es muss aber berücksichtigt werden, dass gerade die Ausdehnung des Gebietes für den Muskelsinn beim Hunde und beim Menschen eine sehr verschiedene ist. Beim Hunde deckt sich dasselbe mit der sogenannten psychomotorischen Region, während beim Menschen der Muskelsinn vor allem im Parietallappen lokalisiert ist. 
Tabelle IV.

Ableitung von der psychomotorischen Region (links).

\begin{tabular}{|c|c|c|c|c|c|c|c|c|c|c|}
\hline \multirow{3}{*}{ 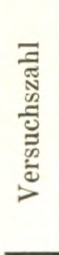 } & \multicolumn{5}{|c|}{$\begin{array}{c}\text { Reizung der rechten Kleinhirn- } \\
\text { hemisphäre }\end{array}$} & \multicolumn{5}{|c|}{$\begin{array}{l}\text { Reizung der linken Kleinhirn- } \\
\text { hemisphäre }\end{array}$} \\
\hline & \multirow{2}{*}{$\begin{array}{c}\text { Ge- } \\
\text { reizt } \\
\text { wurde } \\
\text { mal }\end{array}$} & \multicolumn{3}{|c|}{$\begin{array}{l}\text { Negativwerden der } \\
\text { Grosshirnrinde }\end{array}$} & \multirow{2}{*}{ 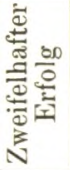 } & \multirow{2}{*}{$\begin{array}{c}\text { Ge- } \\
\text { reizt } \\
\text { wurde } \\
\text { mal }\end{array}$} & \multicolumn{3}{|c|}{$\begin{array}{l}\text { Negativwerden der } \\
\text { Grosshirnrinde }\end{array}$} & \multirow{2}{*}{ 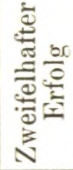 } \\
\hline & & mal & $\begin{array}{l}\text { in } \\
\text { Proz. }\end{array}$ & $\begin{array}{l}\text { Grösse der } \\
\text { Ablenkung } \\
\text { in Skalent. }\end{array}$ & & & mal & $\begin{array}{l}\text { in } \\
\text { Proz. }\end{array}$ & $\begin{array}{l}\text { Grösse der } \\
\text { Ablenkung } \\
\text { in Skalent. }\end{array}$ & \\
\hline 1 & 13 & 8 & 61, & $5-15$ & 2 & 8 & 4 & 5 & $5-16$ & 2 \\
\hline 2 & 10 & 4 & & $5-1$ & 4 & & & & $7-9$ & \\
\hline 3 & 9 & 3 & & $5--11,5$ & 2 & 9 & 0 & & & 2 \\
\hline 4 & 6 & 2 & 33 & $6-9,5$ & 3 & 6 & 2 & 33 & 9 & 2 \\
\hline 5 & a) 9 & 6 & 66,7 & $4-9$ & & & & & & \\
\hline "र & b) 4 & 3 & 75 & $5-11,5$ & 1 & 8 & 5 & & $7-7,5$ & \\
\hline 6 & 11 & 5 & 38 & $5-8$ & & & 2 & & $5-6$ & 1 \\
\hline 7 & 9 & 3 & 33 & $4,5-6$ & 3 & 9 & 3 & & $5-11$ & 9 \\
\hline 8 & 15 & 8 & 53 & $3-7$ & 3 & 9 & 4 & & $3,5-7$ & 2 \\
\hline 9 & 6 & 2 & 33 & $2-4$ & 1 & 7 & 1 & 14,2 & 6 & 3 \\
\hline & 11 & 2 & 18 & $5-10$ & 2 & & 0 & & & \\
\hline 11 & 6 & 1 & 16,7 & 10 & & 6 & 1 & 16,7 & 3 & \\
\hline
\end{tabular}

von Reizen vom Grosshirn zum Kleinhirn für die normale Funktion des letzteren belangreicher sei, als die ungekehrte Reizzuleitung für die Funktion des Grosshirns und dass die sensorischen Erregungen eher und leichter vom Grosshirn zum Kleinhirn als umgekehrt zufliessen. Jedoch möchten wir - obwohl wir zu einer solchen Annahme entgegen der Ansicht mancher Autoren geneigt sind - aus diesen hier mitgeteilten Versuchen keine definitive Schlussfolgerung ziehen. Man könnte auch gegen eine solche Schlussfolgerung eventuell mancherlei Einwände geltend machen wollen. Auf die möglichen Einwände selbst resp. deren Widerlegung wollen wir hier um so weniger eingehen, als wir hoffen, demnächst dieser Frage auf anderem Wege näher zu treten.

In zwei (von den drei) Versuchen, in denen bei Reizung der Kleinhirnrinde Aktionsströme von der Grosshirnrinde häufiger erhalten wurden, wurde hintereinander und abwechselnd von der gewöhnlichen, d. i. von der psychomotorischen Region und zum Vergleich von der Gegend hinter derselben abgeleitet.

Es zeigte sich nun in diesen Fällen, dass bei Reizung der Kleinhirnhemisphäre die Ableitung von der psychomotorischen Region Aktionsströme häufiger zum Vorschein kommen lässt, als bei Ableitung von dem nach hinten von dieser Region gelegenen Rinden- 
Tabelle V.

Reizung der Kleinhirnhemisphäre.

\begin{tabular}{c|c|c}
\hline \multicolumn{2}{|c}{ Auftreten der Aktionsströme bei } & Ableitung \\
\hline $\begin{array}{c}\text { von der } \\
\text { psychomotorischen } \\
\text { Region }\end{array}$ & $\begin{array}{c}\text { von der } \\
\text { hinteren Rinden- } \\
\text { partie B }\end{array}$ & $\begin{array}{c}\text { nochmals von der } \\
\text { psychomotorischen } \\
\text { Region }\end{array}$ \\
\hline $66,6 \%$ & $31 \% \%$ & $50 \%$ \\
$75 \%$ & $16,5 \%$ & $50 \%$
\end{tabular}

bezirk, obwohl der Ableitung von letzterer Gegend eine erfolgreichere Ableitung von der psychomotorischen Region voranging und auch nachfolgte (Tab. V). Ist auch in dieser Serie die Anzahl der. Versuche mit abwechselnder Ableitung von der psychomotorischen und der hinter derselben befindlichen Region eine sehr geringe und an und für sich zu keiner Schlussfolgerung berechtigende, so gewährt es doch ein Analogon zum Verhalten in der ersten Versuchsserie, wo für das Erhalten von Aktionsströmen von der Kleinhirnrinde eine Reizung gerade der psychomotorischen Region sich erfolgreich zeigte. 


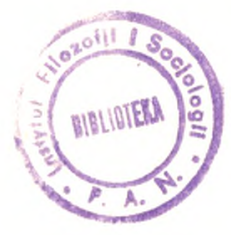

http://rcin.org.pl/ifis 
http://rcin.org.pl/ifis 


\section{PAN 11349}

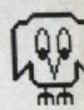

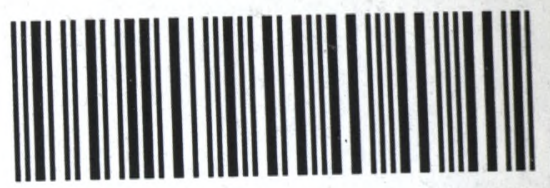

